\title{
28 Research Square \\ Observational Study on Treatment of SRD-Type Diabetic Macular Edema With Aflibercept
}

\author{
Yanlei Hao \\ Qilu hospital, Shandong University \\ Yuan Tao \\ Jinan 2nd People's Hospital \\ Yang Li \\ Huanghe Hospital \\ Jundong Wang \\ Feixian County Hospital \\ Shuting Li \\ Qilu hospital, Shandong University \\ Hong Wang ( $\nabla$ dr.wanghong@163.com ) \\ Qilu hospital, Shandong University

\section{Weiguo Li} \\ Qilu hospital, Shandong University
}

\section{Research Article}

Keywords: serous retinal detachment (SRD), diabetic macular edema (DME), IL-6, hyperreflective dot (HRD), central foveal thickness (CFT)

Posted Date: August 12th, 2021

DOI: https://doi.org/10.21203/rs.3.rs-799123/v1

License: () (i) This work is licensed under a Creative Commons Attribution 4.0 International License.

Read Full License 


\section{Abstract}

To examine the changes before and after intravitreal injection of Aflibercept in patients with SRD-type DME compared with non-SRD-type DME patients. Fifty eyes with SRD-type DME and 50 eyes with nonSRD-type DME were treated with Aflibercept ( 2 mg, $3+P R N)$. The concentration of IL- 6 in aqueous humor was measured before treatment, at 3 and 6 months after treatment. The changes in VA, CFT, and HRDs were observed before treatment and at 1, 2, 3, 4, 5, and 6 months after treatment. The VA in the two groups was improved after treatment $(P<0.05)$. the CFT and the number of HRDs in the two groups after treatment were decreased compared to that before treatment (all $P<0.05$ ). Moreover, the concentration of IL- 6 in the aqueous humor at 3 and 6 months after treatment was reduced compared with that before treatment $(P<0.05)$. This data suggests that inflammation had an important role in DME occurrence, and the occurrence of SRD is closely related to inflammation. Intravitreal injection of Aflibercept can improve the visual acuity and macular edema in patients with DME.

\section{Introduction}

Diabetic macular edema (DME) is an important cause of diabetic retinopathy that can seriously endanger visual acuity. Its occurrence is associated with various factors, including hypoxia and oxidative stress, upregulation of VEGF, disruption of the blood-retinal barrier (BRB), retinal vascular leukocyte stasis, pericyte loss, and vascular hyperpermeability.[1,2] DME is mainly divided into three types: diffuse macular edema, cystoid macular edema, and serous retinal detachment (SRD) type macular edema. Previous studies have reported that the concentrations of VEGF, IL-6, and IL-8 in the vitreous fluid of SRD-type DME patients are elevated, thus suggesting that inflammation has an important role in the pathogenesis of SRD-type DME patients.[3,4] Some experimental studies have also shown that retinal inflammation occurs in a relatively early process of the diabetic process, and it precedes vascular dysfunction and neuronal degeneration. $[1,4]$ Although the exact mechanism through which the release of cytokines leads to inflammation remains unclear,[4] retinal microglial activation has been considered to be mainly responsible for the initial inflammatory response.

The aim of this study was to investigate the changes of HRDs in the retina and the changes of IL- 6 concentration in the vitreous fluid before and after treatment in patients with diabetic macular edema, as well as the correlations between SRD and BCVA, IL-6, and HRDs.

\section{Results}

\section{Visual acuity}

Before treatment, the visual acuity in SRD-type DME patients was significantly lower than that in nonSRD-type DME patients $(P<0.05)($ Table 1$)$. After treatment, the visual acuity in the two groups at 1, 2, 3, 4,5 , and 6 months was improved compared to before treatment $(p<0.05)$; there were no significant differences in the visual acuity between the two groups at $1,2,3,4,5$, and 6 months $(P>0.05)$. 


\section{Central foveal thickness (CFT)}

Before treatment, the central foveal thickness in SRD-type DME patients was significantly higher compared to non-SRD-type DME patients $(P<0.05)$ (Table 2). After treatment, the CFT in the two groups at $1,2,3,4,5$, and 6 months was decreased compared with that before treatment $(p<0.05)$; there were no significant differences in CFT between the two groups at 1, 2, 3, 4, 5, and 6 months after treatment $(P>$ 0.05).

\section{Hyperreflective dots (HRDs)}

Before treatment, the number of HRDs in SRD-type DME patients was significantly higher than that in non-SRD-type DME patients $(P<0.05)$ (Table 3). After treatment, the HRDs in the two groups at 1, 2, 3, 4, 5 , and 6 months were decreased compared with that before treatment $(p<0.05)$. At 1, 2, 3, 4, 5, and 6 months after treatment, the number of HRDs in SRD-type DME patients was higher than that in non-SRDtype DME patients $(P<0.05)$.

\section{Changes in the concentration of IL-6 in the aqueous humor of the two groups before treatment and at 3 and 6 months after treatment}

Before treatment, the concentration of IL- 6 in the aqueous humor of SRD-type DME patients was significantly higher than that of non-SRD-type DME patients $(P<0.05)$ (Table 4). The concentration of IL-6 in the aqueous humor at 3 and 6 months after treatment was decreased compared with that before treatment $(P<0.05)$. There were no significant statistical differences in the concentration of IL-6 in the aqueous humor between the two groups at 3 and 6 months after treatment $(P>0.05)$.

Table 4

Changes in IL- 6 concentration in aqueous humor of the two groups before treatment and at 3 and 6 months after treatment

\begin{tabular}{|llllllll|}
\hline IL-6 & $\begin{array}{l}\text { Before } \\
\text { treatment }\end{array}$ & $\begin{array}{l}\text { 3 months } \\
\text { after } \\
\text { treatment }\end{array}$ & $\mathbf{t}$ & $\mathbf{P}$ & $\begin{array}{l}\text { 6 months } \\
\text { after } \\
\text { treatment }\end{array}$ & $\mathbf{t}$ & $\mathbf{P}$ \\
\hline $\begin{array}{l}\text { SRD-type } \\
\text { group }\end{array}$ & $\begin{array}{l}17.90 \pm \\
4.97\end{array}$ & $9.05 \pm 2.21$ & 11.8453 & 0.0000 & $8.84 \pm 2.19$ & 12.1444 & 0.0000 \\
\hline $\begin{array}{l}\text { Non-SRD- } \\
\text { type } \\
\text { group }\end{array}$ & $9.77 \pm$ & $8.28 \pm 1.89$ & 3.218 & 0.0017 & $8.39 \pm 1.74$ & 3.0497 & 0.0029 \\
\hline $\mathrm{t}$ & 2.94 & & & & & & \\
$\mathrm{P}$ & 10.5298 & 1.9681 & & & 1.1974 & & \\
\hline
\end{tabular}


There were correlations between BCVA and CFT, IL- 6 concentration, and the number of HRDs in the SRDtype group $((r)=0.68715290, t=11.8511, P=0.0000 ;(r)=0.35894478, t=4.8187, P=0.0000 ;(r)=$ $0.31945515, t=4.2241, P=0.0000)$; there were correlations between CFT and IL- 6 and CFT in the SRDtype group $((r)=0.39670535, t=5.4150, P=0.0000 ;(r)=0.39358289, t=5.3646, P=0.0000)$; there was no correlation between IL- 6 and HRDs in the SRD-type group $((r)=0.14738071, t=1.8671, P=0.0638)$. There was a correlation between BCVA and CFT in the non-SRD-type group $((r)=0.42876497, t=6.1699, P=$ 0.0000); there were no significant correlations between BCVA and IL- 6 concentration and HRD number in the non-SRD-type group $((r)=0.07056863, t=0.9197, P=0.1795 ;(r)=0.10206093, t=1.3338, P=0.0920)$; there were correlations between CFT and both IL- 6 and CFT in the non-SRD-type group $((r)=0.24560918$, $t=3.2938, P=0.0006 ;(r)=0.24159766, t=3.2367, P=0.0007)$; there was no significant correlation between IL-6 and HRDs in the non-SRD-type group $((r)=0.08207804, t=1.0706, P=0.1429)$.

\section{Discussion}

Serous retinal detachment (SRD) is defined as an anatomical separation between the neurosensory retina and the subfoveal retinal pigment epithelium. While the pathogenesis of SRD remains unclear, diabetic retinopathy in patients may lead to spillage of lipids and proteins when the blood-retinal barrier is disrupted. Exuded proteins and cells do not pass through the ELM when the ELM is functioning, but when the ELM is affected, the fluid accumulated in the outer retina enters the subretina together with lipids, proteins, and inflammatory cells, thus causing serous retinal detachment.[5,6] Kim et al also suggested that fluid movement caused by the passage of a hyperpermeable choroid through a dysfunctional RPE also causes the development of SRD.[7] In our study, SRD-type DME patients before treatment had worse visual acuity compared with non-SRD-type DME patients, thus suggesting that it may be related to the damage of ELM. Consequently, we need to further observe and study the effect of ELM integrity on visual acuity, while the visual acuity in both groups was significantly improved after treatment, and there was no significant statistical difference in visual acuity between the two groups.

Some scholars have found that intravitreal VEGF, IL-6, and IL-8[4] concentrations are elevated, as revealed by analysis of the concentrations of cytokines in the vitreous fluid of patients with SRD-type DME. Higher IL- 6 concentration is strongly associated with the presence of SRD, which implies that inflammation has an important role in the development of this subtype.[3] IL-6 is the factor that is most significantly associated with the presence of SRD. It may be a proinflammatory cytokine 19 , regulated by nuclear factor-K $\beta$.[8-10] In the SRD-type DME, disruption of the external membrane is accompanied by cell damage, which attracts the entry of scavenger cells into the retina and produces more IL-6.[11] Another reason may be the migration and accumulation of activated microglia into the outer segments of the retinal layers, which may produce excess IL- 6 and lead to fluid accumulation in the subretina. IL- 6 can also promote the secretion of VEGF, and in their study, SHOZO SONODA et al. found that the concentration of intraocular VEGF was significantly correlated with the level of IL-6.[3] In this study, the concentration of IL- 6 in the aqueous humor of SRD-type DME patients was significantly higher than that of non-SRD-type DME patients $(P<0.05)$, thus further confirming the role of inflammation in SRD-type DME. After treatment, the concentration of IL-6 in the aqueous humor of both groups was significantly 
reduced, with no statistical significance between the two, thus suggesting that Aflibercept can inhibit macular edema through anti-inflammation.

At the same time, it has also been found that the SRD-type DME is associated with an increase in HRD and significant functional impairment of the macula.[11] HRD on SD-OCT is considered a clinical sign of the accumulation of activated microglia in the retina[12] and has been associated with severe inflammatory responses.[13] Our experiment revealed that the number of HRDs in the retina of patients with and without SRD increased, which was consistent with previous studies.[14] The number of HRDs in the retina of SRD-type DME patients at baseline was higher than that in the retina of non-SRD-type DME patients $(P<0.01)$, and although the number of HRDs in both groups significantly decreased after treatment compared with before $(P<0.01)$, the number of HRDs in the retina of SRD-type DME patients was still higher than that in the retina of non-SRD-type DME patients $(P<0.01)$, which further confirmed the role of inflammation in SRD-type DME patients.

The level of IL- 6 in aqueous humor and the number of HRDs in the retina of SRD-type DME patients were significantly higher than those in non-SRD-type DME patients, thus suggesting that inflammation had an important role in the occurrence of DME and that the occurrence of SRD was closely related to inflammation. After intravitreal injection of Aflibercept, the visual acuity and macular edema were improved. The level of IL- 6 in the aqueous humor and the number of HRDs in the retina was significantly reduced. These results suggest that the level of IL- 6 in the aqueous humor and the number of HRDs in the retina of DME patients can be used as an inflammatory marker in DME patients.

\section{Methods}

\section{General data}

This prospective study included a total of 100 patients (100 eyes) with diabetic macular edema diagnosed with OCT in our hospital between August 2018 and January 2020. These patients were divided into SRD-type DME group and non-SRD-type DME group with 50 patients (50 eyes) each, according to the mode of edema. Inclusion criteria were the following: (1) patients with DME diagnosed by OCT with the stable general condition; (2) patients with the binocular disease were randomly selected, and only one eye was included in the observation. Exclusion criteria were: (1) poor imaging quality caused by vitreous hemorrhage, cataract, and other refractive media turbidity; (2) cataract surgery within 6 months; (3) antiVEGF therapy or retinal laser photocoagulation was performed; (4) macular edema caused by fundus diseases such as retinal vein occlusion and wet age-related macular degeneration.

\section{Methods}

Patients in both groups were treated with intravitreal injection of Aflibercept $2 \mathrm{mg}$. The $3+$ PRN mode was used. Before treatment, and at 3 and 6 months after treatment, aqueous humor was collected for intraocular fluid detection. The concentration of IL-6 in aqueous humor was measured. The best-corrected visual acuity (BCVA) results were converted to LogMAR for statistical processing. Central foveal thickness 
(CFT) was defined as the average thickness from the inner boundary of the retina to the outside on $1 \mathrm{~mm}$ tomography of the center of the macula. The measurements were taken 3 times by the same experienced technician and then were averaged. HRD was calculated using the OCT 5 line raster scanning mode with a linear scan centered on the fovea, $6 \mathrm{~mm}$ in length, and $0.25 \mathrm{~mm}$ in spacing, by manually counting the high reflection points and then summing the high reflection points on the five scanning lines. This study was approved by the Ethics Review Committee of Qilu Hospital, Shandong University and adhered to the tenets of the Declaration of Helsinki. Informed consent was obtained from all participants and their legal guardians and informed consent was obtained to publish the information in an online open access publication.

\section{Outcome measures}

Changes in best-corrected visual acuity (BCVA), central foveal thickness (CFT), and hyperreflective dots (HDRs) in each group before treatment and at 1, 2, 3, 4, 5, and 6 months after treatment and changes in intravitreal IL- 6 before treatment, and at 3 months and 6 months after treatment were observed in patients.

\section{Statistical analysis}

SPSS 20.0 software was used for statistical processing. Measurement data were expressed as $\overline{\mathrm{x}} \pm \mathrm{S}$. Group design t-test was used for different intergroup data. Paired design t-test was used for intragroup data before and after treatment. $\mathrm{P}<0.05$ was considered statistically significant.

\section{Declarations}

\section{Acknowledgments}

None

\section{Author contributions}

YL.H, H.W, WG.L and Y.T conducted the research conception and design, and drafted the research manuscript. Y.L and JD. W participated in the data collection. ST. L and WG. L carried out the Analysis and interpretation of data.YL. H, H.W and Y.T participated in the design and perfomed statistical analysis. All authors reviewed the final manuscript.

\section{Funding}

None

\section{Competing interests Statement}

The author(s) declare no competing interests.

\section{Data availability}


The datasets used and/or analyzed during the current study are available from the corresponding author on reasonable request.

\section{Ethics declarations}

This study was approved by the Ethics Review Committee of Qilu Hospital, Shandong University and adhered to the tenets of the Declaration of Helsinki.

\section{Consent to participate and publish}

Informed consent was obtained from all participants and their legal guardians and informed consent was obtained to publish the information in an online open access publication.

\section{References}

1. Ascaso, F. J., Huerva, V. \& Grzybowski, A. The role of inflammation in the pathogenesis of macular edema secondary to retinal vascular diseases. Mediators Inflamm. 2014, 432685 (2014).

2. Duh, E. J., Sun, J. K. \& Stitt, A. W. Diabetic retinopathy: current understanding, mechanisms, and treatment strategies. JCl insight. 2, (2017).

3. Sonoda, S., et al. Retinal morphologic changes and concentrations of cytokines in eyes with diabetic macular edema. Retina (Philadelphia, Pa.). 34, 741-748 (2014).

4. Sonoda, S., et al. Correlation between reflectivity of subretinal fluid in OCT images and concentration of intravitreal VEGF in eyes with diabetic macular edema. Investigative ophthalmology \& visual science. 54, 5367-5374 (2013).

5. Marmor, M. F. Mechanisms of fluid accumulation in retinal edema. Documenta ophthalmologica. Advances in ophthalmology. 97, 239-249 (1999).

6. Ota, M., et al. Optical coherence tomographic evaluation of foveal hard exudates in patients with diabetic maculopathy accompanying macular detachment. Ophthalmology. 117, 1996-2002 (2010).

7. Kim, J. T., Lee, D. H., Joe, S. G., Kim, J. G. \& Yoon, Y. H. Changes in choroidal thickness in relation to the severity of retinopathy and macular edema in type 2 diabetic patients. Investigative ophthalmology \& visual science. 54, 3378-3384 (2013).

8. Rizzolo, L. J., Peng, S., Luo, Y. \& Xiao, W. Integration of tight junctions and claudins with the barrier functions of the retinal pigment epithelium. Progress in retinal and eye research. 30, 296-323 (2011).

9. Simó, R., Villarroel, M., Corraliza, L., Hernández, C. \& Garcia-Ramírez, M. The retinal pigment epithelium: something more than a constituent of the blood-retinal barrier-implications for the pathogenesis of diabetic retinopathy. Journal of biomedicine \& biotechnology. 2010, 190724 (2010).

10. Sorrentino, F. S., Allkabes, M., Salsini, G., Bonifazzi, C. \& Perri, P. The importance of glial cells in the homeostasis of the retinal microenvironment and their pivotal role in the course of diabetic retinopathy. Life sciences. 162, 54-59 (2016). 
11. Vujosevic, S., et al. Diabetic Macular Edema With and Without Subfoveal Neuroretinal Detachment: Two Different Morphologic and Functional Entities. American journal of ophthalmology. 181, 149155 (2017).

12. Vujosevic, S., et al. HYPERREFLECTIVE RETINAL SPOTS AND VISUAL FUNCTION AFTER ANTIVASCULAR ENDOTHELIAL GROWTH FACTOR TREATMENT IN CENTER-INVOLVING DIABETIC MACULAR EDEMA. Retina (Philadelphia, Pa.). 36, 1298-1308 (2016).

13. Lee, H., Jang, H., Choi, Y. A., Kim, H. C. \& Chung, H. Association Between Soluble CD14 in the Aqueous Humor and Hyperreflective Foci on Optical Coherence Tomography in Patients With Diabetic Macular Edema. Investigative ophthalmology \& visual science. 59, 715-721 (2018).

14. Vujosevic, S., et al. Imaging retinal inflammatory biomarkers after intravitreal steroid and anti-VEGF treatment in diabetic macular oedema. Acta ophthalmologica. 95, 464-471 (2017).

\section{Tables}

Due to technical limitations, table 1 to 3 docx is only available as a download in the Supplemental Files section.

\section{Supplementary Files}

This is a list of supplementary files associated with this preprint. Click to download.

- Table1to3.docx 